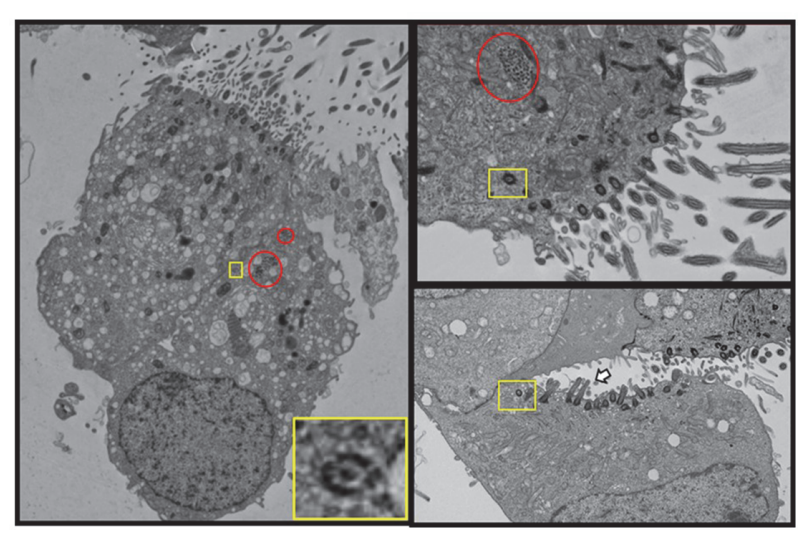

Abstract S55 Figure 1 Transmission Electron Micrographs of ciliated epithelial cells 3months- 1 year post-COVID. Red circles represent microtubular subunits assembling during ciliogenesis, the white arrow shows short regenerating cilia. Yellow boxes surround intracytoplasmic cilia seen in $90 \%$ post COVID cases and suggestive of a defect in ciliogenesis

score was $3.0 \pm 0.6$ compared to $2.4 \pm 0.6$ for controls $(\mathrm{p}<0.01)$. Percentage of goblet cells in post-COVID patients and controls was similar, but there was a significant lack of ciliation of the epithelium post-COVID $(p<0.01)$. No significant improvement was seen In 14 patients with a second sampling point3-5 months later. Ultrastructural analysis revealed evidence of regeneration of ciliated epithelial cells such as the presence of microtubular subunits and basal bodies in the cytoplasm as well as shortened cilia (figure 1).

Ciliary beat amplitude per second was significantly reduced compared to controls $(60.2 \pm 19.2 \mu \mathrm{m} / \mathrm{s}$ vs $78.5 \pm 8.9 \mu \mathrm{m} / \mathrm{s})$ $(\mathrm{p}<0.0001) .29 \%$ individuals post-COVID had some rotating cilia, usually a specific sign for Primary Ciliary Dyskinesia. Ultrastructural analysis revealed $17 \%( \pm 11 \%)$ cilia had defects, these included defects of the central pair of microtubules, in keeping with a rotational beat pattern. 90\% cases had cells with intracytoplasmic cilia, suggestive of epithelial inflammation and dysfunctional ciliogenesis. This was seen in $<1 \%$ controls.

No significant difference was seen between community, hospitalised, or critical care cases.

Conclusion Epithelial disruption and defects in epithelial regeneration persist for up to 1 year post infection with SARS-CoV-2, irrespective of initial illness severity. Persisting inflammation, or a failure of repair, are possible pathological processes for further exploration.

\section{S56 MEASURES OF INFLAMMATION, COMPLEMENT ACTIVATION AND COAGULATION IN PATIENTS WITH COVID-19}

${ }^{1} \mathrm{~L}$ Wiffen, ${ }^{1} \mathrm{~T}$ Brown, ${ }^{1} \mathrm{M}$ Chauhan, ${ }^{1} \mathrm{~L}$ Fox, ${ }^{1} \mathrm{~S}$ Glaysher, ${ }^{1} \mathrm{~S}$ Elliott, ${ }^{1} \mathrm{~L} \mathrm{D}$ 'Cruz, ${ }^{2} \mathrm{M}$ Nunn, ${ }^{3} \mathrm{~T}$ Higenbottam, ${ }^{1} \mathrm{AJ}$ Chauhan. ${ }^{1}$ Portsmouth Hospitals University NHS Trust, Portsmouth, UK; ${ }^{2}$ Akari Therapeutics, London, UK; ${ }^{3}$ Faculty of Pharmaceutical Medicine, London, UK

\subsection{6/thorax-2021-BTSabstracts.62}

Introduction Severe Acute Respiratory Syndrome Coronavirus2 (SARS-CoV-2) has resulted in a global pandemic of unprecedented magnitude causing a disease that varies widely in nature and severity ranging from asymptomatic carriers to lifethreatening illness requiring ICU support. Excessive immune activation is believed to underpin the pathophysiology, however, despite the identification of treatments which target this (Dexamethasone, Tocilizumab) there remains significant morbidity and mortality suggesting other pathobiological mechanisms are at play including the complement cascade.

Methods We compared the clinical, physiological \& biological characteristics including levels of inflammation, complement activation and coagulation of 52 patients with confirmed or clinically suspected COVID-19, stratified based on their degree of respiratory failure (Mild: FiO2 $\leq 0.4$; Moderate: FiO2 $>0.4$ \pm NIV support (HFNO/CPAP) or Severe: mechanical ventilation) and compared to 20 age and co-morbidity matched

Abstract S56 Table 1 Table to show measures of complement activation, inflammation and coagulation in patients with COVID-19 stratified by disease severity

\begin{tabular}{|c|c|c|c|c|c|c|c|}
\hline & Mild & Moderate & Severe & Overall P-Value & Mild vs Mod* & Mild vs Sev* & Mod vs Sev* \\
\hline Number (\%) & 30 & 28 & 9 & - & - & - & - \\
\hline CRP (mg/L) & $75.5[28.5,117.25]$ & $93.5[72,143.5]$ & $60[34,157.5]$ & NS & NS & NS & NS \\
\hline Ferritin (ug/L) & $426[290.5,847.5]$ & $728[381.25,1071.5]$ & $857[443,1607.5]$ & NS & NS & NS & NS \\
\hline PCT (ug/L) & $0.08[0.06,0.19]$ & $0.16[0.09,0.49]$ & $0.19[0.12,0.8]$ & 0.019 & NS & 0.046 & NS \\
\hline LDH (U/L) & $708.5[523.5,903]$ & $830[569,1122]$ & $1037[927.5,1086]$ & 0.008 & NS & 0.006 & NS \\
\hline Platelets $(10 * 9 / \mathrm{L})$ & $220[174.75,328.75]$ & $255[203.5,335]$ & $292[209.5,329.5]$ & NS & NS & NS & NS \\
\hline INR & $1.1[1.08,1.23]$ & $1.1[1.1,1.2]$ & $1.1[1.1,1.15]$ & NS & NS & NS & NS \\
\hline APTR & $0.9[0.9,1.0]$ & $0.9[0.9,1.0]$ & $1.0[0.95,1.10]$ & NS & NS & NS & 0.038 \\
\hline D-dimer (ugFEU/ml) & $0.56[0.38,0.95]$ & $0.74[0.55,1.52]$ & $0.85[0.54,19.2]$ & NS & NS & NS & NS \\
\hline Fibrinogen ( $g / L)$ & $4.45[4.05,5.22]$ & $4.7[4.3,6.38]$ & $4.3[3.9,6.0]$ & NS & NS & NS & NS \\
\hline Thrombin-AT III Complex ug/L) & $4.75[2.65,12.13]$ & $8.8[5.3,12]$ & $14.3[6.9,40.7]$ & 0.045 & NS & NS & NS \\
\hline Prothrombin Fragment $1 \& 2(\mathrm{pMol} / \mathrm{L})$ & $275[164.5,380.5]$ & $311[163,492]$ & $301[258,709]$ & NS & NS & NS & NS \\
\hline CH50 (U Eq/ml) & $123.4[101.75,174.7]$ & $113.4[88.68,153.43]$ & $114.7[74.6,158.9]$ & NS & NS & NS & NS \\
\hline Complement C5a (ng/ml) & $29[21.5,36]$ & $36.5[25.5,48.75]$ & $68[39.5,122.5]$ & $<0.001$ & 0.038 & 0.001 & NS \\
\hline Complement C5 (mg/L) & $270[235.25,290.5]$ & $263[235.25,279]$ & $276[220,299.5]$ & NS & NS & NS & NS \\
\hline Complement C3 (g/L) & $1.5[1.25,1.77]$ & $1.48[1.33,1.71]$ & $1.56[1.16,1.73]$ & NS & NS & NS & NS \\
\hline SC5b-9 complex (ng/ml) & $1070.46[836.41,1632.17]$ & $1725.48[1092.62,2403.3]$ & $2392.66[1245.68,5145.88]$ & 0.006 & NS & 0.019 & NS \\
\hline Complement Fragment $\mathrm{Bb}(\mu \mathrm{g} / \mathrm{ml})$ & $0.2[0.15,0.27]$ & $0.25[0.17,0.3]$ & $0.29[0.2,0.43]$ & NS & NS & NS & NS \\
\hline Complement (3a ng/ml) & $296.88[244.33,345.22]$ & $325.88[248.33,484.03]$ & $460.23[282.49,652.1]$ & NS & NS & NS & NS \\
\hline
\end{tabular}


volunteers. In the event of deterioration with escalation of severity category repeat samples were obtained.

Results Patients with COVID-19 were more likely to be male (67\% vs $20 \%(\mathrm{HC}) ; \mathrm{p}<0.001)$, older $(64.4 \pm 16.7$ vs $47.7 \pm$ $13.5 ; \mathrm{p}<0.001)$ have a greater BMI $(32.3 \pm 6.6$ vs $27.9 \pm$ $5.1 ; \mathrm{p}=0.01)$ and be never-smokers $(60 \%$ vs $30 \% ; \mathrm{p}=0.001)$. We demonstrated a hyperinflammatory and pro-coagulative state in all patients with COVID-19. All measures of complement activity were significantly higher in patients with COVID-19, including levels of C5a (HC 13[7,21] vs Covid-19 $35[24,43] ; \mathrm{p}<0.001$ ) and SC5b9-complex (HC 654[419, 1120] vs Covid-19 1452[970, 2170]; p<0.001) which both increased with disease severity and were statistically significantly different between mild and severe disease. SC5b9-complex was significantly higher in patients who deteriorated from moderate to severe disease (1393 [1019, 1986] vs 2116 [958, 4538]; $\mathrm{p}=0.03)$.

Discussion Our findings demonstrated increased levels of complement activity in patients with COVID-19, particularly in those patients requiring non-invasive and mechanical ventilation and those patients that deteriorate requiring increasing ventilatory support. The complement cascade is a key player in protective immunity against pathogens, with its activation orchestrating key immunoprotective and anti-inflammatory effects. Increased activation of the complement cascade may contribute to the dysregulated and destructive inflammatory response that leads to multi-organ failure and our findings suggest a potentially important treatment target for COVID-19.

\section{S57 EXPRESSION OF THE SARS-COV-2 RECEPTORS ACE2 AND TMPRSS2 IN THE RESPIRATORY TRACT OF CHILDREN AND ADULTS}

AG Nuttall, C Hedrich, C Semple, P Losty, R Shukla, J McPartland, S Williams, S Northey, P McNamara. University of Liverpool, Liverpool, UK

\subsection{6/thorax-2021-BTSabstracts.63}

Background Angiotensin-converting enzyme 2 (ACE2) receptors and the serine protease co-factor TMPRSS2 are cellular receptors/co-factors for SARS-CoV2, allowing viral entry into host cells. Children, when infected with SARS-CoV2, generally present with mild disease, and particularly milder lower respiratory tract symptoms. One hypothesis to explain this phenomenon is differential expression of ACE2 and TMPRSS2 in the respiratory tracts of children and adults.

Aims To investigate ACE2 receptor and TMPRSS2 expression in upper/lower respiratory tracts of children and adults without COVID disease.

Methods Nasal brushings from obtained from children of different ages (3 months -15 years) undergoing routine elective surgery, and from volunteer adults (20-61 years). Nasal epithelial ACE2 and TMPRSS2 mRNA expression was analysed by PCR.

Post-mortem lung tissue from children and adults without COVID-19 was stained to identify ACE2 and TMPRSS2 protein expression by immunohistochemistry (IHC). Each sample was digitalised using Philips Digital Pathology Solutions software, with three alveolar and three bronchial screen-grab images obtained at $\mathrm{x} 40$ magnification, and analysed using Image $\mathrm{J}$.

Results Nasal ACE2 and TMPRSS2 mRNA expression in children and adolescents $(n=12)$ and adults $(n=26)$ was similar. Immunohistochemical lung tissue ACE2 and TMPRSS2 protein

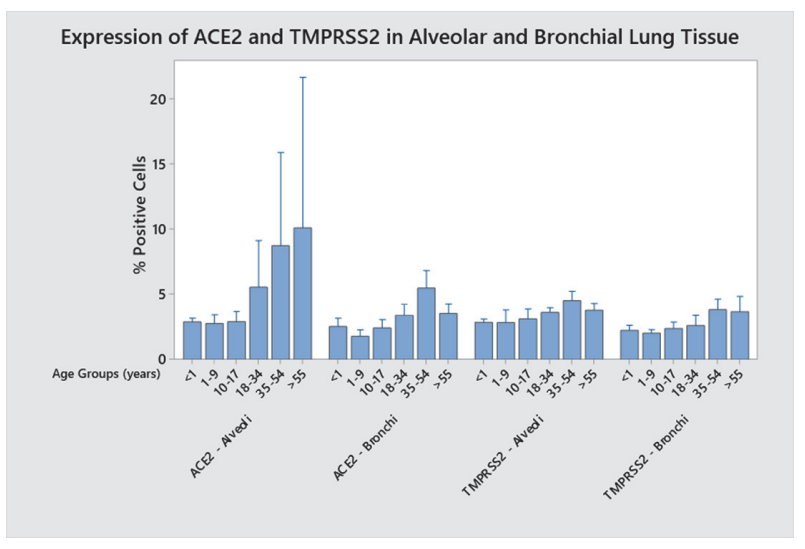

Abstract S57 Figure 1 Bar chart with error bars displaying mean $\%$ positive cells for each age group for alveolar and bronchial ACE2 and TMPRSS2

expression in 38 subjects $(<1 \mathrm{yr} n=9,1-9 \mathrm{yrs} n=4,10-17 \mathrm{yrs}$ $\mathrm{n}=6,18-34 \mathrm{yrs} \mathrm{n}=7,35-54 \mathrm{yrs} \mathrm{n}=7,>55 \mathrm{yrs} \mathrm{n}=5$ ) was significantly greater in alveolar than bronchial sections. In children, ACE2 and TMPRSS2 expression was detected in only 2-3\% of cells in alveolar and bronchial tissue. Alveolar ACE2 receptor expression was significantly greater in adults and appeared to increase with age. Adult alveolar ACE2 receptor expression was highly variable, being detected in some specimens in $\sim 30 \%$ of cells.

Conclusion ACE2 and TMPRSS2 expression is similar in upper airways of children and adults, likely indicating that both groups are equally susceptible to SARS-CoV2 infection. In contrast, expression of both these receptors/co-factors is greater in adult lower airways of adults, and particularly for ACE2 receptor in alveolar tissue. In some adults, ACE2 receptor was detected in up to a quarter of alveolar cells, potentially explaining why some adults are so susceptible to lower respiratory tract disease.

\section{Treatment choices in cystic fibrosis and bronchiectasis: what works and when}

\section{S58 TRIPLE CFTR MODULATORS IMPROVE SINO-NASAL AND LARYNGOPHARYNGEAL REFLUX SYMPTOMS IN PEOPLE WITH ADVANCED CYSTIC FIBROSIS LUNG DISEASE}

${ }^{1} \mathrm{~S}$ Shakir, ${ }^{1} \mathrm{C}$ Echevarria, ${ }^{1} \mathrm{~S}$ Doe, ${ }^{2} \mathrm{M}$ Brodlie, ${ }^{2} \mathrm{C}$ Ward, ${ }^{2} \mathrm{~S} J$ Bourke. ${ }^{1}$ Adult Cystic Fibrosis Centre, Royal Victoria Infirmary, Newcastle upon Tyne, UK; ${ }^{2}$ Translational and Clinical Research Institute, Newcastle University, Newcastle upon Tyne, UK

\subsection{6/thorax-2021-BTSabstracts.64}

Background Triple CFTR modulator therapy (elexacaftor/tezacaftor/ivacaftor) improves lung function, weight, exacerbation rates and quality of life in people with Cystic Fibrosis. CF is a multisystem disease and there is increasing interest in the extrapulmonary effects of CFTR modulators. Chronic rhinosinusitis and gastroesophageal reflux are common in people with CF and cause a high level of sino-nasal and laryngopharyngeal symptoms. We assessed the effect of triple CFTR modulator therapy on these symptoms in a cohort of patients with advanced CF lung disease.

Method In a prospective study, we used the Sino-Nasal Outcome Test (SNOT), the Reflux Symptom Index (RSI) and the 